\title{
Taking an (Embodied) Cue From Community Health: Designing Dementia Caregiver Support Technology to Advance Health Equity
}

\author{
Connie Guan \\ Computer Science and Engineering, \\ UC San Diego \\ San Diego, CA, USA \\ cguan@ucsd.edu
}

\author{
Anya Bouzida \\ Computer Science and Engineering, \\ UC San Diego \\ San Diego, CA, USA \\ abouzida@ucsd.edu
}

\author{
Ramzy M. Oncy-Avila \\ Computer Science and Engineering, \\ UC San Diego \\ San Diego, CA, USA \\ roncyavi@ucsd.edu
}

\author{
Sanika Moharana \\ Computer Science and Engineering, \\ UC San Diego \\ San Diego, CA, USA \\ sanikamoharana@gmail.com
}

\author{
Laurel D. Riek \\ Computer Science and Engineering, \\ UC San Diego \\ San Diego, CA, USA \\ lriek@ucsd.edu
}

\begin{abstract}
Dementia affects $>50$ million worldwide, causing progressive cognitive and physical disabilities. Its caregiving burden falls largely onto informal caregivers, who experience their own health problems, and face tremendous stress with little support-all exacerbated during COVID-19. In this paper, we present a new caregiver support perspective, where the lenses of health equity and community health can shape future technology design. Through a 1.5 year long, in-depth research process with dementia community health workers, we learned how caregiving support technology can reflect key concepts in dementia community health practice. This paper makes two contributions: 1) We propose employing embodied cueing, such as imitation or action mimicry, as a communication modality that can align technology with community caregiving approaches, promote agency in people with dementia, and relieve caregiver burden, and 2) We suggest new avenues for HCI research to advance health equity in the context of dementia technology design.
\end{abstract}

\section{CCS CONCEPTS}

- Applied computing $\rightarrow$ Health informatics; • Human-centered computing $\rightarrow$ Participatory design.

\section{KEYWORDS}

dementia, design, health equity, dementia caregiving, social determinants of health, robots, community health

ACM Reference Format:

Connie Guan, Anya Bouzida, Ramzy M. Oncy-Avila, Sanika Moharana, and Laurel D. Riek. 2021. Taking an (Embodied) Cue From Community Health: Designing Dementia Caregiver Support Technology to Advance Health Equity. In CHI Conference on Human Factors in Computing Systems

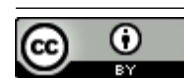

This work is licensed under a Creative Commons Attribution International 4.0 License.

CHI '21, May 8-13, 2021, Yokohama, Japan

(C) 2021 Copyright held by the owner/author(s).

ACM ISBN 978-1-4503-8096-6/21/05

https://doi.org/10.1145/3411764.3445559
(CHI '21), May 8-13, 2021, Yokohama, Japan. ACM, New York, NY, USA, 16 pages. https://doi.org/10.1145/3411764.3445559

\section{INTRODUCTION}

Many researchers in HCI and related fields have been exploring new technologies and design methods to support people with dementia (PwD) [23, 32, 68, 75]. Much of this work has focused on non-pharmacological treatments, such as reminiscence therapy, where PwD recall life events to improve psychological well-being, or sensory stimulation, which involves stimulating one or more of the PwD's senses to encourage engagement and positive emotions $[5,30,40,43,50,59,69,116]$. Within the gerontology community, the focus has shifted from primarily designing for PwD, to also considering the importance of how technology can support informal caregivers [63]. These are unpaid family members or friends who provide daily support to PwD, usually in the PwD's home, including support for activities of daily living (ADLs), like grooming, eating, and mobility, and with cognitive functioning tasks (instrumental ADLs (IADLs)), such as problem solving, scheduling, and managing medication and finances.

Dementia caregiving is particularly difficult due to the unique cognitive and physical changes in a P $\mathrm{PD}$ that necessitate increasingly greater assistance from the caregiver as the condition progresses [82]. Many informal caregivers face heavy financial and emotional burdens as they manage the physical and cognitive changes in their loved ones over the course of many years [17, 79]. Informal caregivers are often older adults themselves, likely managing their own chronic health conditions in addition to those of the PwD. Furthermore, the burden and stress of dementia caregiving places informal caregivers at a higher risk of developing additional comorbidities, negatively impacting their mental, cognitive, and/or physical health [94].

A recent challenge facing informal caregivers and $\mathrm{PwD}$ is the COVID-19 pandemic. Due to the higher likelihood of both being over the age of 65 and having comorbidities, both parties are at higher risk of disease-related morbidity and mortality. In addition, the pandemic has disrupted their access to existing community care ecosystems, including dementia day care facilities, support 
groups, and other community resources, many of which have been suspended due to social distancing policies. While some of these resources have been replaced with video teleconference, due to technology access issues, many community members remain unable to participate [102]. Collectively, these issues have resulted in a high degree of social isolation and negative psychosocial health for informal caregivers and PwD [104]. This situation has highlighted existing weaknesses in dementia care structures, especially regarding access to caregiving resources and support.

Many in the public health community have explored caregiving difficulties and access to caregiving resources as a social determinant of health (SDoH) [35, 107]. SDoH include how mortality, morbidity, health care expenditures, life expectancy, functional limitations, and health status are impacted by factors including socioeconomic status, level of education, physical environment, race, ethnicity, etc. The SDoH model offers a lens to understand how these macro and meso factors affect systemic availability and distribution of health resources [65, 115]. Researchers in HCI have begun to adopt this model to examine how technology access and upstream health interventions can address these social determinants and improve health equity $[96,106]$.

$\mathrm{HCI}$ researchers have also examined barriers to inclusive and accessible dementia technology through critical dementia, a lens that rejects deficit-driven design approaches, and examines dementia's social construction to understand embodied expressions of self and personhood [52,57]. This shift has included a broader scope of participants, including practitioners' perspectives on dementia caregiving, such as art or occupational therapists [23, 57]. Critical dementia explores how researchers can incorporate embodied expressions of self in the design and research process for dementia technology $[42,57,58]$. It has led to further insight into the design of more inclusive dementia technology and methods to engage PwD in participatory research [13, 60, 73].

This prior work provides an excellent framework to approach the design of dementia support technology, but there are still gaps in our understanding of how to design technology to address key $\mathrm{SDoH}$, as situated within the broader scope of community care ecosystems.

In this paper, we report a 1.5-year long partnership with community-based dementia organizations to deeply understand this complex ecosystem of community caregiving, and understand how technology may be situated within it, with an eye toward advancing health equity. We adopt a lens in our work that incorporates concepts from community health, gerontology, and critical dementia to explore the intersection of community caregiving practices and technology for at-home dementia care support for informal caregivers.

Due to COVID-19 restrictions on our research process, including many of our partners' facilities shutting down and substantial hardships faced by informal caregivers, the work we report in this paper focuses on the perspectives of professional caregivers.

In this paper, we present two primary contributions. First, we identify key insights from dementia community caregivers that can be applied to technology design. We discuss how embodied cues, which are non-verbal, embodied action prompts, can be adapted as a communication modality for caregiving technology to promote self-agency for PwD and relieve caregiver burden. Second, we explore new avenues for HCI applications and research to potentially address health inequities in caregiving through community perspectives on technology design. We identify three areas where technology can further health equity to overcome these barriers in dementia care, including: providing access to community resources, supporting caregiver wellness through education, and extending the reach of community and social support systems.

\section{BACKGROUND}

In this section, we provide an overview of current dementia care technologies, describe the role of critical dementia within HCI, and provide an overview of the $\mathrm{SDoH}$ model and its relationship to dementia caregiving.

\subsection{Existing Technology Interventions to Support People with Dementia}

Many commercial technologies are used by informal dementia caregivers. They tend to center around safety, monitoring, reminders, and memory assistance [97]. Safety and monitoring interventions include baby monitors [64], wearables (like talking watches) [4], and GPS-based geofencing technologies. Memory and reminder assistance technologies include spoken reminder machines and lost object locators [4].

However, commercial technology has been criticized for lacking consideration of the needs and perspectives of its users. For example, even for technology co-designed with $\mathrm{PwD}$, the focus tends to be on early stage dementia (or it is otherwise unreported) [98], which may limit its utility across more advanced stages when it is of greater need. Another criticism is that many commercial technology designs stigmatize and exclude PwD by emphasizing their frailty (e.g., large red alert buttons), or by having a high degree of complexity [45].

This deficit-centered framing of dementia is often reflected and perpetuated by commercial technology, where both dementia and aging are framed as a steady depletion of ability, and loss of self [74]. This technosolutionist / deficit-framed model has been criticized by many (c.f. [11]). HCI dementia research has taken an active stance to move away from this model towards more social-relational framing of dementia that challenges the idea that one's cognitive or physical abilities are the sole marker of their personhood [23, 57, 61] (See Section 2.3).

To support this, researchers have included more diverse stakeholders such as informal and professional caregivers, PwD, and others when designing technology to support dementia caregiving $[23,32,48,68,75]$. For example, Sriram et al. [97] conducted a review of informal carers' experiences with assistive technology, and found they expressed mixed views. They enjoy the help that the technology gives, yet worry that they do not challenge PwD cognitively in early stage dementia. They were also concerned technology may strain the relationship between the caregiver and $\mathrm{PwD}$ as the reliance on the technology grows [97].

Dixon and Lazar [23] worked with professional dementia practitioners to explore how to support PwD's engagement in meaningful and purposeful activities. They encourage dementia technology designers to incorporate dementia practitioners' views (albeit in a nuanced way), a philosophy we embrace in our work. 


\subsection{Robotic and Embodied Technologies to Support Dementia Caregiving}

Socially assistive robots, or robots that provide assistance through social interactions, are frequently used in dementia care [87]. These robots provide sensory experiences to $\mathrm{PwD}$, and are often zoomorphic in appearance to mimic animal therapy (without allergies and scratching) [54]. Paro, a robotic seal, is the most well-studied example, but others include $\mathrm{NeCoRo,} \mathrm{a} \mathrm{robotic} \mathrm{cat,} \mathrm{and} \mathrm{Aibo,} \mathrm{a}$ robotic dog $[66,100,109]$. These robots can reduce symptoms of anxiety, agitation, and depression in PwD [76], and also help facilitate human-human interaction [90]. Others have built functional robots in the context of dementia caregiving, such as to help with wayfinding, reminders, and locating items [7, 24, 95, 105, 113].

Many studies in HRI suggest that the physical embodiment and presence of robots makes them exceptionally well-suited to be effectual deliverers of health interventions compared to virtual agents [1, 20, 26, 46, 47, 88, 119]. Embodied robots can support engagement, facilitate interactive presence [99], and provide contextualized and socially situated cues [101]. These interactions can then be personalized to adapt to the abilities of the PwD $[55,56]$. They can support interaction in a range of new ways that extend interventions into the physical world far beyond the current state of practice [117]. This is well-aligned with recent work in the dementia literature, showing that embodied activities such as personalized art, music, or dance therapies are particularly effective means to engage PwD [6]. These embodied therapies are frequently used to soothe PwD, especially in later stages when verbal language can become difficult $[49,108]$.

\subsection{HCI Research and Dementia}

The traditional biomedical perspective frames dementia as a series of losses and impairments that steadily erode the PwD's sense of self [53]. This can cause PwD to be stigmatized and isolated, and treated in ways that reduce their sense of agency and personhood. However, social-relational models of dementia challenge the idea that one's cognitive or physical abilities are the sole marker of personhood [85]. Researchers within the HCI community have made great strides in technology design for dementia that challenge the biomedical view $[12,29,36,41,110,112,114]$. For example, Wallace et al. [110] draw on a person-centric model of dementia, which proposes that personhood dynamically emerges out of social interactions. The authors examined designing for personhood in dementia relationships through extensive work with Gillian, a woman living with dementia, and her husband John. This work resulted in the design of digital jewelry that invites others to engage with Gillian in relation to her personhood rather than her dementia diagnosis.

Another model, critical dementia, explores expressions of personhood in dementia through embodiment, context, and emotional and sensorial experience [51, 52, 57]. This lens is well-suited to HCI, aligning with shifts in the field away from cognitivist views, toward more holistic perspectives of interaction and meaning-making [57]. For example, HCI researchers have explored how PwD can interact with the world in meaningful ways through art, music, or dance $[16,75]$. Others have examined the role of technology in validating a sense of agency and personhood for PwD [31].
HCI work has also applied these socio-relational models of dementia to caregiving relationships and design research. Researchers in $\mathrm{HCI}$ have shown how $\mathrm{PwD}$ are often placed in more passive roles within care relationships as their caregiver takes over care tasks, financial responsibilities, or household decisions [34, 44]. This work critically examines how HCI researchers can create more inclusive design spaces by incorporating opportunities for mutual and meaningful social recognition into the design process $[28,29]$.

\subsection{Dementia Caregiving and Social Determinants of Health}

In our research, we worked with community health workers and found that in addition to the critical dementia lens outlined above, we also needed to include a community health frame to understand the community health perspective our participants took to dementia care. We draw from the public health field to critically analyze the $\mathrm{SDoH}$ that affect caregiving. The US Centers for Disease Control (CDC) define $\mathrm{SDoH}$ as the "conditions in the places where people live, learn, work, and play that affect a wide range of health risks and outcomes" [33]. These factors can include: socioeconomic status, education, physical environment, race, ethnicity, internet access, community and social support networks, or early parental support $[2,10]$.

The SDoH model recognizes that psychosocial factors such as stress, depression, low social standing, isolation, or low self-esteem also have a significant impact on an individual's health outcomes throughout their life [21, 67]. The World Health Organization (WHO) incorporates SDoH into a life course approach to health, which incorporates how social, economic, and political factors influence one's socio-economic position, which in turn affect health outcomes.

The SDoH model can be realized across three levels of organization- macro, meso, and micro [2]. At the macro level are structural factors that generate socioeconomic hierarchies and govern access to resources in society [80]. A few examples are public and political policy, macroeconomic policy, and sociocultural norms. Where an individual falls within these hierarchies can be indicated by attributes such as income, gender, social class, or ethnicity [2, 80].

While these macro structural mechanisms are the source of social stratification and health inequity, this inequity is instantiated through meso, or intermediary, level factors. Meso level determinants are often at the level of communities or institutions such as workplace or university policies, community access to healthy food or clean water, and neighborhood quality. Meso level determinants also include psychosocial factors such as stressful environments, social relationships or quality of support networks. Inequities at the meso level are both the result of, and reinforced by macro-level structural mechanisms. Finally, at the micro level are individual factors such as genetics or lifestyle factors $[2,80]$.

Within dementia care, a SDoH model reveals how informal caregivers' increased stress puts them at risk for negative health outcomes $[15,118]$. However, public health interventions can address meso-level SDoH to reduce these psychosocial stressors by providing informal caregivers with access to strong community resources, social support services, caregiver training and education, and transportation [103]. For example, Lorenz et al. [64] found that informal 


\begin{tabular}{|c|c|c|}
\hline $\begin{array}{l}\text { Design Probe } \\
\text { Prototyping }\end{array}$ & $\begin{array}{l}\text { Reflection with } \\
\text { Design Probe }\end{array}$ & $\begin{array}{l}3 \text { Envisioning Future } \\
\text { Technology }\end{array}$ \\
\hline $\begin{array}{l}\text { Engaged in an iterative prototyping } \\
\text { process to collaboratively develop a } \\
\text { design probe. }\end{array}$ & $\begin{array}{l}\text { Conducted interviews with design probe } \\
\text { to understand how technology might be } \\
\text { incorporated into dementia caregiving }\end{array}$ & $\begin{array}{l}\text { Explored how technology can support } \\
\text { caregiving goals for informal caregivers }\end{array}$ \\
\hline
\end{tabular}

Figure 1: We engaged in a 1.5-year long, in-depth research process with dementia community health workers.

caregivers are largely unaware of available dementia assistive care technologies, and often opt to repurpose other widely available technologies like baby monitors and music players into their care routines.

As others have argued, there are many opportunities for HCI researchers to incorporate knowledge of the $\mathrm{SDoH}$, in order to build more inclusive and accessible technologies, which will help further health equity $[10,37,96,106]$. This is particularly true within the context of dementia caregiving [22]. This framework contextualizes and frames the public health issues community health workers engage with, such as informal caregiver education or limited community resources. For example, while critical dementia was essential in understanding our participants' discussions of embodied communication, this discussion was in the context of a need for caregiver education. We needed the SDoH lens to understand the role and importance of such community education within dementia care.

\section{METHODOLOGY}

Over the past one and a half years, we have engaged in an in-depth research process with members of multiple dementia community health organizations. Through this process, we sought to understand existing dementia caregiving practices from a community health perspective.

In this paper, we report findings from our work with two community-based dementia non-profit organizations. Both focus on providing support to informal caregivers in different ways.

The first organization is a local chapter of a national Alzheimer's Association, which focuses on dementia awareness, caregiver training, and providing support resources to the community. They are also active in offering support groups and classes to PwD and their caregivers.

The second organization is an adult day care facility that provides affordable and accessible programs for PwD. This facility offers daytime care for PwD, who are still able to live at home, but require additional supervision during the day to give their caregivers respite and an opportunity to work and/or run errands. The facility curates innovative activities based on art and reminiscence therapy to actively engage PwD based on severity and stage. They also provide family support and case management, along with community education programs.

\subsection{Participants}

Our participants held a variety of different roles across the two organizations described above. For clarity we provide the following definitions of how we define these roles in this paper. A social worker is someone who offers support, dementia resources, and training to people affected by dementia. Practitioners include occupational therapists, activity coordinators, and art therapists, and professional caregivers who work to help PwD. An informal or family caregiver is a friend or family to the PwD who provides care without compensation.

All participants had past or current experience in professional dementia caregiving. Over half have at least 20 years experience deeply embedded in the dementia care community, directly working with informal caregivers across a range of settings, including athome support. They are heavily involved in caregiver education, focusing on home care. Participants' ages ranged from $20 \mathrm{~s}-50$ s (four people did not provide ages), and all were women. We employed pseudonyms for both our participants and any $\mathrm{PwD}$ they mentioned to preserve privacy, while representing their individuality (see Table $1)$.

\subsection{Design Probe and Goals}

We iteratively created a design probe in collaboration with members of the dementia community. The aim of our research was to gain insight on how to better design for strengths within PwD. Therefore, in our interviews with the design probe, we framed our questions in such a way that participants would speculate possible use cases of our probe. These responses inform the actual use of potential technology, while informing the design process as well.

Others have done similar work where speculative narratives, or design fictions, are used to inform the design process [78, 92, 93]. In our case, we used a design probe rather than a narrative to explore speculative technology.

\subsection{Research Process}

Figure 1 depicts our three-phased research process, which we describe in detail below.

3.3.1 Phase 1: Design Probe Prototyping. In this phase, we were interested in exploring the design space of community dementia care practices. The goal of this phase was to prototype a design probe that could evoke conversation and speculation about future design of dementia technology.

We worked with two social workers from the local Alzheimer's Association chapter. We engaged in ethnographic observations and multiple rounds of interviews and prototyping to understand how we could integrate technology design with community best practices. We asked questions such as, "What are some pain points in dementia caregiving?", "What tips are there for informal caregivers 


\begin{tabular}{|c|c|c|c|c|c|c|}
\hline Pseudonym & Age & Gender & Experience & Organization & Role & Responsibilities \\
\hline Ava & 45 & F & 20 years & Alzheimer's Association & Social Worker & Informal caregiver training and education \\
\hline Julia & 57 & F & 34 years & Alzheimer's Association & Social Worker & Informal caregiver training and education \\
\hline Phoebe & - & F & 2 years & Adult Day Care & Care Practitioner & Plan and coordinate dementia-friendly activities \\
\hline Misa & 48 & F & 30 years & Adult Day Care & Social Worker & Plan and lead dementia education programs \\
\hline Belinda & - & F & 2 years & Adult Day Care & Care Practitioner & Oversee other care staff; Lead activities and care for PwD \\
\hline Catalina & - & F & 1 year & Adult Day Care & Care Practitioner & Lead activities and care of PwD \\
\hline Alicia & - & F & 1 year & Adult Day Care & Care Practitioner & Lead activities and care for PwD \\
\hline
\end{tabular}

Table 1: Demographic information of the dementia health community workers who participated in our research. Participants had a broad range of experiences and perspectives on care. We employ pseudonyms to preserve the privacy of participants.

to make caregiving easier?", or "What settings would you envision informal caregivers using this prototype?".

Based on our contextual research, we chose to focus on mealtimes. We learned that informal caregivers struggle with mealtimes, both for themselves and for PwD, because PwD often need reminders to eat or to continue eating. This in turn makes it difficult for informal caregivers to find time to eat. By providing support during mealtimes, informal caregivers have more time for meaningful interactions with loved ones, or a moment of respite, during a meal.

In this phase we discussed the following four design considerations: 1) familiarity, 2) mimcry, 3) music, 4) durability and cost.

Familiarity: An important design consideration participants suggested was that familiar objects are less distressing for PwD, who may not be comfortable with technology. Additionally, the technology should be embodied within an appropriate object for the context and setting (e.g. a toaster is appropriate in the kitchen).

Mimicry: They also discussed the importance of mimicry or pantomime motions as an ability that PwD retained longer during the course of dementia, so could be useful to be reflected in design.

Music: They also often mentioned music as a means to provide emotional well-being to $\mathrm{PwD}$, and potentially bring a positive aspect of joy to the task. A strong awareness for rhythm and music is retained in $\mathrm{PwD}$, even in advanced stages, and can be used to initiate and maintain tasks such as eating and bathing.

Durability and Cost: Another consideration was that it was imperative for the technology to be durable, as PwD can be rough with handling technology (described by participants and in the literature, c.f. [70]). Another high level design goal was to have a low cost, easy-to-build design to make our technology easily accessible to vulnerable populations.

Ultimately, we decided on co-creating a physically embodied robot that reflected these caregiver design considerations. The design probe, Spoonbot, can be seen in Figure 2. The robot's older-style radio design is both familiar to older adults and appropriate for the context of use, as it plays music on a tabletop during mealtimes. It plays music familiar to western audiences (top Billboard hits from the 1950s) in an attempt to take advantage of music and reminiscence therapies. The robot mimics eating by holding a spoon and engaging in basic self-feeding arm movements. This takes advantage of PwD's ability to mimic eating motion through pantomime. It has a low cost, ruggedized design with suction cups, to attach to the table, and secure bindings for its internal wirings.
3.3.2 Phase 2: Reflection with Design Probe. In the second phase we were interested in understanding how technologies like Spoonbot might be incorporated into dementia caregiving practice. We conducted semi-structured interviews with two social workers and three professional caregivers using Spoonbot from Phase 1 as a design probe to inspire speculation about technology for dementia care. (See section 3.2)

We split the interview into two stages: 1) feedback on the design probe, and 2) reflections on caregiving practice. We first introduced participants to the design probe and asked for feedback on the probe's design features. Next, we learned more about their role and experiences with dementia caregiving approaches. Using an open-ended interview format, we found that often Spoonbot sparked more interesting insights regarding specific care situations or broader caregiving ideologies. ${ }^{1}$

3.3.3 Phase 3: Envisioning Future Technology. In the third phase, we wanted to take the knowledge gained from the previous phase to explore how technology can support those broader caregiving ideologies amongst informal caregivers. We returned to our initial two co-designers from the first phase and conducted semi-structured interviews and a design brainstorming activity.

We split the interview into three stages. Due to COVID-19 limitations, the interviews were conducted over teleconference. First, we reviewed design probe feedback from Phase 2 with co-designers. Second, we asked questions regarding informal caregiving practices based on what we learned from Phase 2. Finally, we conducted a brainstorming activity where participants were given a common caregiving task (dressing, taking medicine, or bathing) and asked to reflect on Spoonbot and envision how an informal caregiver would use a technology that embodies caregiving approaches learned from Phase 2.

\subsection{Data Collection and Analysis}

We audio-recorded the in-person interviews from Phases 1 and 2. We recorded both audio and video in the teleconference interviews from Phase 3, but extracted only the audio for analysis. We transcribed the data using transcription software, and then reviewed it manually. All interviews were conducted with one participant at a time, except for Phoebe and Misa's interviews in Phase 2, who were interviewed together.

We analyzed the data using thematic analysis, following the process outlined by Braun et al. : initial read through, code generation,

${ }^{1}$ To support reproducibility of our work, all interview questions will be posted on GitHub. 

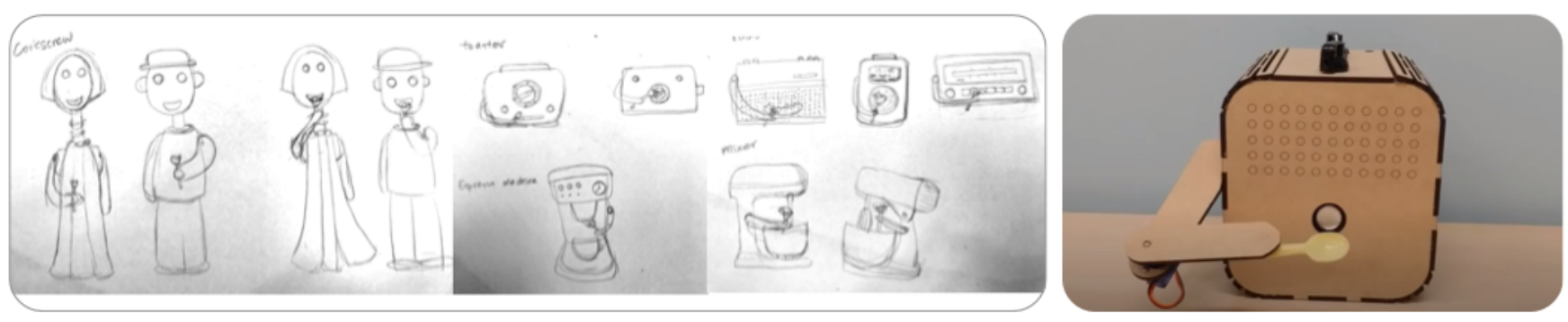

Figure 2: Iterative evolution of our design probe Spoonbot, from early sketches to final prototype, co-designed with a subset of stakeholders. Our original ideas were based on familiar kitchen objects to address feeding and mealtimes. Key design features include: a) Arm which conveys an Eating Motion to leverage pantomime to assist PwD with eating; b) Old-Style Radio Morphology to be a familiar and appropriate object for PwD during mealtimes; c) Playing Music to provide rhythm, help initiate tasks, and support emotional wellbeing; d) Ruggedized Design to be durable to withstand PwD handling items roughly.

theme development, refining, naming, and writing cohesively [14]. Three researchers individually coded the data, and met regularly to discuss. The coders did an initial read through to familiarize with the data and find initial codes. They then conducted an iterative process of generating codes and combining them into themes.

\section{FINDINGS}

Through our interviews, we learned about dementia community health worker's perspectives on informal caregiving and technology. Our key findings are as follows: (1) Participants identified key techniques to support self agency, (2) We recognized interdependent relationships between task assistance and emotional support, 3) Participants discussed the use of embodied cueing in dementia care, and 4) We identified technology roles to support caregiver-PwD dynamics. We discuss these in detail below.

\subsection{Community Health Perspectives on Agency in Caregiving}

As with prior work in the critical dementia community [16, 31, 57, 75], our participants stressed the importance of providing PwD ample opportunities to cultivate self-agency and normalcy. Doing so can be impactful to both PwD and informal caregivers by helping to promote a sense of normalcy in daily life. Here, we describe how dementia community health workers apply these concepts while interacting with PwD.

4.1.1 How Communities of Care Reaffirm Agency in PwD. A main goal of professional caregivers is to promote self-agency in PwD by engaging them in situations that highlight their strengths. Belinda described a common experience during mealtimes where some PwD would take out their wallet to tip her.

"I've gotten tips [...] one gentleman [...] takes out his wallet. He goes 'Hold on. Hold on', and he's struggling, and he gives me a dollar and he's like 'Here you go. Don't spend it all in one place'. I'm like 'No no no. It's okay. It's okay.' "

By allowing the PwD to tip her, she creates a narrative that validates his ability to practice self-agency.
Professional caregivers have noticed that PwD want to be viewed as capable, so, as a caregiver, they provide a space where PwD can contribute. Phoebe describes a PwD who used to be an accountant:

"For the longest time Carol thought she was our accountant. So I would print invoices and she tells me all the time 'You um spend a lot of money.' I say 'I do?' And she goes 'Yes I told you your budget is fifty dollars. What is this?' And then [I am] like 'Oh, I'm so sorry Carol, I'm so sorry.'”

While Carol is not serving as an accountant, professional caregivers still respected and acknowledged Carol's domain expertise, and in doing so promoted her sense of self-agency.

Professional caregivers set PwD up for success when interacting with them, by being aware of the kinds of questions that they are capable or incapable of responding to. Phoebe describes her approach to interacting with PwD: "We can't ask anything where they feel like they're gonna get it wrong [...] and once [the PwD] gets it wrong they go back into like 'Oh my gosh what's wrong with me?' " Professional caregivers are aware of the possibility for negative interactions that may affect the mood of PwD and purposefully manage their interactions to set PwD up for success.

Professional caregivers reinforce a sense of normalcy and personhood for the PwD by designing various activities and events that mirror familiar experiences. For example, Phoebe discussed activities designed to be like going out to a restaurant:

"So I set [up] like an Italian [restaurant] date for them. I served them first their salads, and it was all on like ceramic plates and I wanted them to be real. [...] I made a menu for them to look at. So you kind of give them back that experience [...] a lot of family members don't take them back to restaurants because there's just way too many triggers."

In another activity, Phoebe describes the flower bouquets that PwD made, "[A PwD] made that. They all made that. And I bet you those things could sell for 50 bucks at least. [...] They can do so much with it. Their imagination is still there." These activities serve to recapture experiences which may no longer be accessible for PwD due to their condition. Providing opportunities to re-experience 
eating at a restaurant or creating professional bouquets are opportunities to recognize and validate the inherent personhood of PwD.

\subsection{Combining Task Assistance and Emotional Support}

It is emotionally taxing for an informal caregiver to witness their loved one becoming a stranger and gradually lose recognition of their relationship and memories together. Learning to balance and create separation between a PwD's practical daily needs against their emotional needs is incredibly challenging for an informal caregiver. Considering this immense burden, informal caregivers must learn how to detach their emotions from fulfilling caregiving responsibilities for the PwD. Here we present our findings on how technology can improve the emotional wellbeing of both informal caregivers and PwD by recognizing the emotional factors within dementia caregiving.

4.2.1 Emotional Impact of Caregiving Challenges. While informal caregivers do their best to care for their loved one, they often run into conflict with the PwD when attempting to direct tasks (e.g., to take medication). Julia discussed the dynamic between informal caregivers and $\mathrm{PwD}$ and the potential issues that arise during care, "As adults living with dementia, [they] fight for their autonomy. They become resistant. They don't want to do these things just because [the caregiver] told [them to]." These caregiving tasks are important to manage the health of the PwD, however the PwD may see this constant direction as undermining their autonomy.

Informal caregivers often face challenges when helping a PwD initiate and complete ADLs. This can negatively impact the psychosocial wellbeing of both caregiver and PwD. Ava explains the difficulties of initiating and motivating PwD in caregiving tasks, "When I was talking about helping [a PwD] with initiating a task, [it's] kind of motivating getting [them] into the physical movement of an activity." The difficulty of dementia caregiving for informal caregivers is the high levels of both physical and emotional support that PwD require in simple and repetitive tasks.

Ava further describes challenges caregivers face in adapting to the dynamic nature of dementia symptoms:

"First, sort of figuring out where [the PwD is] at. How confused are they? How disrupted do their motor skills seem to be today? And if today is a hard day for [the PwD] then it's probably gonna involve a lot more physical assistance. Whereas if they're having a higher functioning day, it might be a matter of [just] laying [their] clothes out [near] the bath."

Not only is the caregiving itself difficult, informal caregivers face additional cognitive burden by needing to continuously monitor and adapt to changes in the PwD physical and mental capabilities.

This is compounded by the fact that common verbal methods of communication, like explanation or negotiation, may not be appropriate for PwD and can cause them to be uncooperative. Ava described her advice for a common struggle for informal caregivers when interacting with PwD, "Don't get into the negotiation about, [task timing], or [reasons for doing a task]. That's what [an informal caregiver is] always trying to do-is talk the person into something. Whereas if they just got the task started, 9 times out of 10 it's going to happen." The informal caregivers need to learn new communication styles to make dementia caregiving less stressful.

These challenges during caregiving assistance for ADLs are not only frustrating and stressful for caregivers, but also impact the mood of the PwD. Ava describes the cycle of frustration and tension she sees between caregivers and $\mathrm{PwD}$, "there [are] a lot of carepartners [that] find themselves engaging in a lot of like senseless arguing [...] Then you have to distract the [PwD] with some kind of an activity that's relaxing and soothing." Difficulties during dementia care tasks cause conflict and agitation that impact the emotional wellbeing of the PwD as well. At this point, the care task evolves from assisting an ADL into managing an emotional response of the PwD, adding additional complexity and burden to caregiving.

4.2.2 Opportunities for Technology to Address Task Support for Emotional Wellbeing. Dementia community health workers saw opportunities for technology to assist informal caregivers and PwD with tasks in order to support greater emotional wellbeing. Julia discussed the draining nature of dementia caregiving, "[the caregivers] need to become the initiator, and that gets hard [...] because basically it's like cheerleading 24/7." Julia suggested Spoonbot could provide an opportunity to provide respite to informal caregivers, "Even if it didn't really prompt the [PwD] to start eating [Spoonbot] might [distract] the person [and with] a few minutes of that person's attention and interest [informal caregivers] can walk away and do something else for a minute." Considering the co-dependent nature of task assistance and emotional support, technology that addresses both factors equally can reinforce the well-being of both the informal caregiver and PwD.

COVID-19 has created an especially challenging environment for PwD and their caregivers. The heightened situation gives us an opportunity to consider creating accessible dementia technology that provides respite and also emotional support for informal caregivers, especially when quarantining in their homes. Julia described the discomfort and isolation caused by COVID-19, and the state of the current caregiving environment, "Now dealing with these incredibly socially isolated [PwD]. Something like [Spoonbot] would be more helpful than ever, just as as a diversion for the [PwD] for a few minutes." This is an opportunity where technology can engage the PwD to provide informal caregivers a moment of respite.

\subsection{Embodied Cueing}

Across all of our interviews, all participants spoke about the benefits of embodied cues within caregiving and technology design.

4.3.1 Embodied Cueing in Caregiving Practice. When discussing effective modes of communication, participants often mentioned that verbal language, if vague or non-concrete, was often unsuccessful. Phoebe explains, "We have [several individuals who are] very high functioning, and once you ask them [a vague question] it will completely throw off the entire thing [...] Because in that moment, they feel like 'I don't know what they're talking about. 


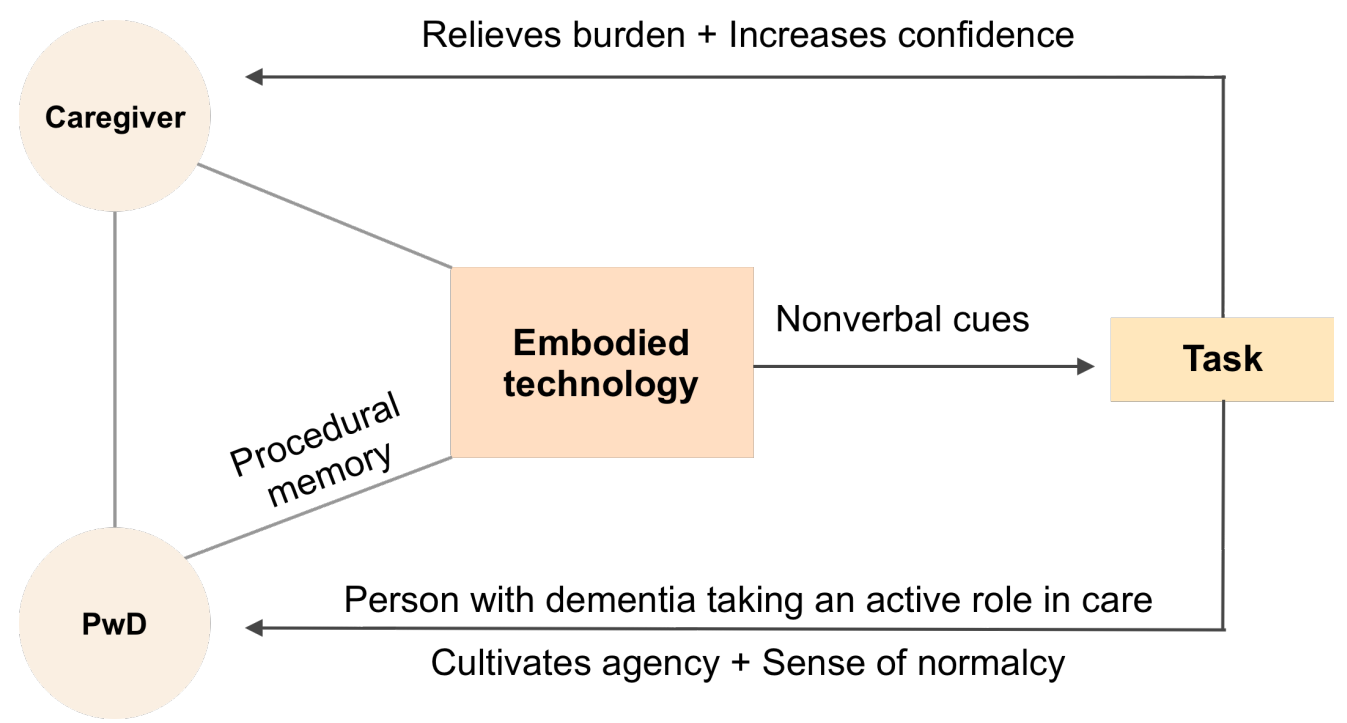

Figure 3: How embodied technologies mediate caregiver-PwD interactions to support emotional well being in care tasks.

What's wrong with me? Now I'm angry.' " It can be difficult for people with moderate to advanced dementia to process abstract verbal language. This can cause frustration especially among PwD who have anosognosia, where they are unable to recognize that they have dementia. This can cause a PwD's confidence to plummet, as they perceive themselves as being "faulty", but are unable to figure out why.

Dementia community health workers also suggested that using embodied cues can help convey meaning. Belinda spoke of a daycare participant who usually needs to be fed and said:

"When we do sit her in groups she [...] see[s] others [and] how they're eating. So then she will grab the spoon and then put it in [...] her mouth, and then [...] a couple seconds later, again, she won't be able to use it, but [...] seeing other participants in front of her that are eating [helped her to] eat."

In this case, the cue of other people eating was able to help this PwD remember how to eat, even if it was just for a moment.

Professional caregivers employ visual cueing as a communication mechanism. Phoebe explains, "It just makes it easier when there is a visual, it eliminates [questioning if you are] communicating [...] or explaining [something] incorrectly." This helps reduce confusion resulting from the ambiguities of language and verbal instructions.

Embodied cues can be a more effective caregiving technique than reminders. As Ava explains:

"with Alzheimer's type of dementia [...] a reminder just doesn't mean anything [...] they need some kind of either physical or even just a visual prompt to kind of get the starter button going right. Once they're moving, they can generally take that task and run with it [...] but their brain kind of forgot how to initiate it."

She suggested ways to use this sequencing to help caregivers assist with ADL tasks like taking medication, “It's more the mechanics of getting it down right $[. .$.$] maybe modeling the sequencing of$ getting the pill into your hand, [and] taking the drink of water." This demonstrative nature of visual and embodied cues can prompt PwD to perform the corresponding sequence of actions.

4.3.2 Incorporating Embodied Cueing in Technology Design. Participants suggested several ways to incorporate embodied cueing in technology design (See Figure 3).

During our design probe activity, participants brainstormed how technology can help PwD and their caregivers engage during caregiving tasks. Ava gave an example of how technology could support a PwD going to the restroom, "If a robot could demonstrate to the person with dementia the act of walking to the restroom, I think that would be a game changer. Something that's not a reminder, it's showing them." Technology that can show PwD how to do an action in a way that is visual and embodied is helpful to PwD by helping them more actively initiate ADLs.

Participants also highlighted incorporating non-verbal sound features as auditory sentiment as a mode of communication and interaction understood by PwD. Julia explains, "Language often gets garbled up with dementia [...] in terms of being able to understand and process, but noises are easier, you know, [...] if you're going [unhappy sounding hum] that's not a happy noise, right? [happy sounding hum] that is."

\subsection{Caregiver-PwD-Technology Triad Roles and Interactions}

During our interviews, we explored the roles that a technology intervention like Spoonbot could play in supporting the caregiverPwD dynamic (See Table 2). The two main avenues of support were encouraging and mediating caregiver-PwD interaction, and helping the caregiver engage the PwD in task initiation and completion.

4.4.1 Embodied Technology to Support and Encourage Positive Interactions. In supporting $\mathrm{PwD}$, technology can mediate positive 


\begin{tabular}{|c|c|c|c|c|}
\hline & \multicolumn{2}{|c|}{ Mediate Interactions } & \multicolumn{2}{|c|}{ Task Assistance } \\
\hline & Motivator & Respite Provider & Facilitator & Educator \\
\hline What it is/does & Promotes positive interactions & $\begin{array}{l}\text { Engages } \mathrm{PwD} \text { and allows the care- } \\
\text { giver some respite }\end{array}$ & $\begin{array}{l}\text { Facilitates task completion for } \\
\text { PwD directly through movement, } \\
\text { music, etc }\end{array}$ & $\begin{array}{l}\text { Demonstrates effective caregiv- } \\
\text { ing strategies that the caregiver } \\
\text { can learn and perform }\end{array}$ \\
\hline How it supports caregivers & $\begin{array}{l}\text { Gives focus to effective caregiv- } \\
\text { ing strategies }\end{array}$ & $\begin{array}{l}\text { Relieves stress, promotes aregiver } \\
\text { well being }\end{array}$ & $\begin{array}{l}\text { Reduces caregiver strain by aid- } \\
\text { ing PwD in specific tasks }\end{array}$ & $\begin{array}{l}\text { Increases caregiver confidence in } \\
\text { helping with PwD ADLs }\end{array}$ \\
\hline How it supports PwD & $\begin{array}{l}\text { Supports emotional wellbeing } \\
\text { and connection to caregiver }\end{array}$ & $\begin{array}{l}\text { Engages and provides emotional } \\
\text { support }\end{array}$ & $\begin{array}{l}\text { Helps with movement sequenc- } \\
\text { ing in task completion }\end{array}$ & $\begin{array}{l}\text { Affords improved care quality } \\
\text { and thus emotional well-being }\end{array}$ \\
\hline
\end{tabular}

Table 2: Roles for technology to support dementia caregivers and PwD.

interaction between the caregiver and PwD. When speaking on how Spoonbot may be used in caregiving, Ava explains, "A reference point is a good way to put it, I think [...] Spoonbot's not feeding the person. Spoonbot is there to be prompt, a point of conversation." In other words, the robot shouldn't be replacing interaction, instead, it can serve as a tool to be used by the PwD-caregiver dyad, and provide a more valuable point of conversation.

Caregivers can use technology as a motivator to relieve that burden of caregivers. Julia explains, "So Spoonbot becomes the motivator, becomes the initiator of activities, and it allows the carepartner to put that onus on Spoonbot a little bit rather than themselves." This changes the dynamic from "You have to do this because it's good for you", to "That thing the robot is doing is neat, why don't we do that". This enables the caregiver to offload some of the stress of doing the task onto the robot, which acts as a partner in the interaction.

Participants also discussed how Spoonbot or a similar technology could act in a role of respite provider. Informal caregivers can have their PwD engage with a robot, so they can disengage for a bit. Ava explains, "Caregivers get tired of trying to think of ways to distract and engage and tell stories or sing songs [...] if the robot [can be] a resource for the carepartner to kind of lean on, 'look at this little thing that's eating' [...] that distraction, and sort of companion ability is invaluable." The companionship the robot provides to the $\mathrm{PwD}$, in turn, will provide the caregiver a moment for themselves. Julia furthers this idea saying, "It's to keep the person engaged for a period of time. So that the caregiver [can] kind of take a bit of a timeout."

4.4.2 Embodied Technology to Support Caregiving ADLs. A facilitator role is a task-specific role technology can adopt, where the technology facilitates task-based actions by the PwD. The focus is placed on the interaction between the robot and PwD, and how caregiving techniques can be adapted to help through the embodied technology itself. Ava speaks on the potential benefits of a Spoonbot-like robot, "It just sort of jump start[s] triggering a person's procedural memory, to get them started with this task on their own." Spoonbot uses pantomime to encourage the PwD to eat. More broadly, pantomime can be used to facilitate many other potential movements.

Technology can also facilitate task completion through the use of music. Ava said, "Music is incredibly powerful as a motivator, but also just getting people's [...] motor movement [...] in line with what you're trying to do [...] So if I want someone to start doing something, I start doing it, and I start humming a rhythm to that, and I'm very likely to get them to start mimicking me, to start joining me." The technology serving in a facilitation role can use music as a tool that draws attention to the pantomime, to then encourage the PwD to join in.

Technology can also serve in the role of an educator for informal caregivers, by showing successful caregiving strategies. Informal caregivers can learn and gain confidence in effective caregiving techniques and apply them to other tasks. Ava explains, "More broadly is that I think it could teach something to the family carepartner, right? If it's effective, it allows that family carepartner to see the person that they care for being able to do something on their own." She continues, "So I think the real benefit is that if [Spoonbot] works, if it gets the person engaged, and they see [that the PwD starts] following along and doing [the movement], it's gonna show that family caregiver ways they could translate that kind of like modeling to other tasks.” Technologies like Spoonbot may help informal caregivers to move away from doing tasks for a PwD, and instead model techniques caregivers can use to do a task with the PwD.

\section{DISCUSSION}

In our findings, we described how dementia community health workers approach caregiving and support for informal caregivers. Participants envisioned ways technology can support the emotional wellbeing for both caregivers and PwD, which some scholars argue is one of the most important aspects of caregiver support [63, 79]. Drawing from these findings, we describe considerations for future dementia technology design, along dimensions including affording embodied communication and dementia-friendly communication styles, and supporting caregiver and PwD wellness. We then take a community health perspective to suggest three ways technology designers can advance health equity for families with dementia, including by providing access to community resources, supporting caregiver wellness through education, and extending the reach of community and social support systems.

\subsection{Design Considerations}

5.1.1 Non-verbal, embodied communication. All of our participants strongly and repeatedly identified the importance of using embodied cueing during caregiving interactions with $\mathrm{PwD}$. This theme was particularly prominent when envisioning future technologies. Participants described ways technology can convey these cues via visual, tactile, and aural means.

While several commercial technologies employ some of these cues (such as Paro the robotic seal [109]), it may be helpful to 
consider additional technology designs that can both afford cueing modalities and support ADLs. For example, our design probe Spooonbot provides embodied cueing and pantomime to trigger procedural memories related to eating. Participants suggested similar types of cueing could be used for other common day-to-day activities, like bathing, teeth brushing, etc. By helping PwD perform tasks in ways that are well-matched to their cognitive abilities, embodied technology can reduce frustration and improve PwDcaregiver relationships.

Designing for embodied cueing embeds community care practices into the design of dementia technology. We have found that our participants already incorporate non-verbal communication strategies in their caregiver education programs. These communication strategies are based on community approaches of leveraging strengths in PwD to aid care tasks. By drawing from these community care practices, we can design technology interventions that are attuned with broader dementia caregiving practices.

Our findings also highlight the importance of dementia technology being adaptable and adjustable to interact with the unique memory changes that PwD undergo. In the early stages of dementia, prospective memory, or the memory of planned future actions, may be mostly intact. Here, designs which employ reminders or alert-based technologies can be helpful to support PwD with IADLs. However, as the disease progresses and prospective memory becomes weaker, reminder technologies become less effective and can cause tensions and frustration. In particular, there may be a mismatch between the technology's affordances, the informal caregiver's expectations, and the PwD's capabilities. Instead, as dementia progresses, technology design can shift to adopt more embodied interaction styles to leverage strengths in PwD's procedural memory. We expect that this kind of adaptivity can better support both PwD and their caregivers.

5.1.2 Simultaneously Designing for Emotional Wellness Alongside Task Assistance. When designing technology for dementia care, it is important for researchers and designers to pay attention to the social-emotional factors of caregiving. A large part of day-to-day caregiving involves both supporting the emotional wellness of the PwD and managing the logistics of complex care tasks. Technology designed to take advantage of PwD's strengths in caregiving tasks support this social-emotional wellness by validating the agency and personhood of the PwD. Rather than framing PwD as passive recipients of care, technology can help them become active participants in their own care.

We illustrate this through our findings related to embodied cueing in dementia technology design. Embodied cues rely on strengths of PwD's procedural memory. This strength-driven design approach allows for greater agency and emotional wellbeing for PwD. It can provide respite to informal caregivers by making it easier to employ caregiving best practices. Finally, it changes the caregiver-PwD dynamic by presenting $\mathrm{PwD}$ as active participants alongside their caregivers instead of passive recipients of care.

When designing dementia care technology, it is critical for designers to recognize and reflect on the relationship between the caregiver and PwD. Most currently available commercial caregiving technology is designed primarily for the $\mathrm{PwD}$, or their caregiver.
However, we found that these care relationships are often tightly interdependent and interwoven with emotional context. Rather than designing for individual PwD or individual caregivers, it may be beneficial to tailor designs for the pre-existing relationship between PwD and caregivers. For example, how can dementia technology support spouse-spouse, parent-child, or other categories of PwDcaregiver relationships?

5.1.3 Designing to Embody Community Care Practices. When designing dementia care technology, HCI research can embed community care practices directly into technology. It is important to consider the needs of individual end users, but also how technology can further goals of the community. For example, caregivers and PwD need ways to facilitate communication, but the way technology supports this communication can align with broader community care practices. Design that embodies practices of community care couples the design of technology to macro-level missions and policies within community health.

In our work with community health workers, we found that the way they envisioned future technologies for dementia care were influenced by a community health perspective. Our participants discussed embodiment and embodied communication, key concepts in critical dementia and HCI. But, unlike previous work, they focused on how these concepts from critical dementia can inform design to address broader issues of health equity and $\mathrm{SDoH}$ within the community. These perspectives not only shaped the above design considerations, but also informed how to use them to address SDoH. In the following section, we draw on these community perspectives and a SDoH lens to provide more details on how technology can address health equity.

\subsection{Addressing Health Equity}

In our work, we identified ways that technology can help overcome barriers to achieving health equity. These include: providing access to community resources, supporting caregiver wellness through education, and extending the reach of community and social support systems. We propose key principles to consider and several pitfalls to avoid in dementia caregiving technology design.

5.2.1 Providing Access to Community Resources. COVID-19 has highlighted opportunities for technology to address barriers surrounding accessibility of community support resources. In our work, participants discussed how COVID-19 has disrupted access to community support resources, and exacerbated issues of isolation and stress among informal caregivers and PwD. Resources like support groups or caregiver training are typically provided in person. This creates issues of accessibility due to distance, transportation, and lack of disability accommodations.

While video teleconferencing technologies have been helpful for some populations to overcome these barriers, many informal caregivers do not have access to broadband internet, and often have low technology literacy, making most of these technologies inaccessible [10]. Furthermore, for PwD, prior HCI research has criticized an over-reliance on screens as they can be inaccessible due to some dementia-related symptoms [45]. Instead, we propose $\mathrm{HCI}$ researchers can improve community resource accessibility by 
extending the reach of community support into homes through embodied technology.

To support this effort, researchers can draw from best practices within the caregiving community, which can be embodied within technology design practice. Here, rather than adopting a micro-level stance centered on the caregiver, e.g., "How can technology address issues in caregiving burden?”, in our work we adopted a macro-level, community-focused perspective, e.g., "How do community practices address issues in caregiving burden, and how can technology make those practices more accessible?". For example, our participants envisioned robots that could use mimcry, a community taught care practice, to support PwD. This approach to caregiving research in $\mathrm{HCI}$ grounds the design of accessible technology directly in context of community practices and needs.

To illustrate this point, we take as an example art therapy, a meaningful activity used in dementia caregiving to promote social engagement and self expression in PwD [23, 58]. Technology to extend accessibility to the home might look like a digital paper notebook, designed with the look and feel of a traditional notebook, paired with a digital art frame that allows PwD to create and share art. When PwD create art in the notebook, it appears in their art frame, but can also be shared digitally to the art frames of other families using the same technology. Capitalizing on an embodied and familiar design (a notebook and frame) and familiar activity (art making and sharing), coupled with some social sharing, helps PwD easily adopt new technologies and connect with other community members. In doing so, this technology helps informal caregivers directly gain benefits of these community practices.

5.2.2 Supporting Caregiver Wellness through Education. The ability for informal caregivers, especially new ones, to receive training in caregiving practice is another $\mathrm{SDoH}$ that impacts emotional wellness for informal caregivers and PwD. Community-based caregiving education can provide practical training on strategies including embodied cueing, dementia-friendly language, and use of clear visual communication modalities, as well as techniques and resources to manage the daily stress of providing care. This education can increase caregiver confidence in navigating logistical and emotional challenges of dementia care.

Participants highlighted how technology can play a key role in helping provide caregiver education. It is important to note that caregiver education technology does not refer solely to online learning platforms, instead we mean technology that can scaffold the act of complex care tasks. Our participants discussed how technology can teach informal caregivers to use embodied cues in their caregiving practice, by providing an example for them to model. This acts as a scaffold to lessen the burden of learning to apply new techniques in an already stressful situation.

Ultimately, the goal is to facilitate stronger social interactions between the caregiver and PwD. Here, technology may be used to incorporate contextual information to provide in-the-moment feedback and suggestions to caregivers. For example, one might envision a pocket-sized robot that demonstrates embodied cues for informal caregivers, a digital scrapbook that provides dementiaappropriate reminiscence prompts, or a digital board that can be customized with relevant visuals to aid communication.
To encourage the use of dementia-friendly communication, a wearable could provide caregivers with dementia-appropriate conversation suggestions, or suggest opportunities for redirection if it notices an increase in decibel level due to arguments. Similar concepts have been successfully employed in other "just in time" adaptive mental and behavioral health technology interventions, including for depression, schizophrenia, and smoking cessation $[9,77,84]$. Here, technology can alleviate the stress of adopting a new communication strategy in the midst of an already stressful care situation. It also acts as an educational tool that provides guidance on effective communication strategies at the moment it is needed so that informal caregivers can learn to use these strategies themselves.

Another example might be a wearable that detects agitation in PwD through heart rate, and changes color accordingly. This reduces the stress of caregiving by providing feedback to caregivers that PwD may struggle to communicate themselves. At the same time it can teach caregivers how to recognize early signs of agitation in PwD and adapt their caregiving approach. Similar technology designs have been employed in the autism community, e.g., physiological sensors to provide social-emotional sensing and expression, and may also prove helpful within a dementia caregiving context [25].

5.2.3 Extending the Reach of Community and Social Support Systems. Through our work it is clear that caregiving does not occur in isolation, rather, it interacts with complex $\mathrm{SDoH}$ such as community and social factors. Earlier, we addressed barriers to accessibility, e.g., "How can technology help more people access art therapy?". Here we look at barriers to wider availability, e.g., "How can technology support more art therapy sessions?". Availability is another facet of accessibility [62] and can be particularly important to address dementia care in rural or other under-resourced communities $[8,72]$. The scope and scale of community dementia resources are limited by restrictions on staffing or funding. To address this barrier, technology can be designed to address pain points in the delivery of community support services.

While these technologies may not directly be used by PwD or caregivers, they provide indirect impact by improving the ability of community systems to provide support. In our work, community health workers discussed Spoonbot in conjunction with their existing curriculum regarding embodied cues. For areas with less robust caregiving education programs, technology could serve as a platform to help other community health workers create educational programs.

Technology that directly assists PwD or caregivers is absolutely necessary and valuable, but technology can also improve systems and processes that support community health. This approach to design can address issues of technosolutionism, by recognizing that technology interventions alone are not sufficient to address complex social issues.

To illustrate this, we provide the example of a dementia support program that provides weekly group reminiscence sessions. These are intended to provide $\mathrm{PwD}$ with social engagement and provide caregivers with respite such as described in Dai et al. [19] . In order to provide personalized sensory cues to evoke reminiscence in participants, the organizer must spend time planning and researching 


\begin{tabular}{|c|c|c|}
\hline $\begin{array}{l}\text { Health Equity in } \\
\text { Dementia Care }\end{array}$ & How Technology can Help & Examples \\
\hline $\begin{array}{l}\text { Access to Community } \\
\text { Resources }\end{array}$ & $\begin{array}{l}\text { Support informal caregiver access to } \\
\text { community resources at home } \\
\text { - Create opportunities for community } \\
\text { care practice outside of physical } \\
\text { community spaces } \\
\text { - Provide more accessible ways of social } \\
\text { engagement and sharing within } \\
\text { community }\end{array}$ & $\begin{array}{l}\text { - Digital paper notebook and frame to } \\
\text { create and share art with others, } \\
\text { promoting self-expression in PwD }\end{array}$ \\
\hline $\begin{array}{c}\text { Support Informal } \\
\text { Caregiver Education }\end{array}$ & $\begin{array}{l}\text { Reduce caregiver cognitive load and } \\
\text { support emotional wellbeing } \\
\text { - Structure care tasks to promote best } \\
\text { practice } \\
\text { - Provide examples for informal } \\
\text { caregivers to learn from }\end{array}$ & $\begin{array}{l}\text { - Caregiver wearable to provide } \\
\text { dementia-appropriate conversation } \\
\text { suggestions, or opportunities for } \\
\text { redirection } \\
\text { - PwD wearable to detect agitation and } \\
\text { offer feedback to caregivers }\end{array}$ \\
\hline $\begin{array}{l}\text { Extend Reach of } \\
\text { Community and Social } \\
\text { Support Systems }\end{array}$ & $\begin{array}{l}\text { Broaden dementia ecosystem of care } \\
\text { - Expand ability of community health } \\
\text { workers to scale support programs } \\
\text { - Consider pain points in delivery of } \\
\text { community support services }\end{array}$ & $\begin{array}{l}\text { - Online digital database to support } \\
\text { community sharing of customizable } \\
\text { activities and materials for community } \\
\text { support programs } \\
\text { - Ubiquitous sensor systems to track } \\
\text { PwD changes and alert care staff }\end{array}$ \\
\hline
\end{tabular}

Table 3: Key areas for HCI technology design to advance health equity in dementia care.

appropriate materials. Other groups may want to start a similar program, but lack the time and expertise to do so.

A technology that could address this might be a digital scrapbook, which can be customized to provide a variety of different sensory cues through sound files, images, or scents through a diffuser. This scrapbook would be connected to an online database where dementia community health workers can upload, share, and search for personalized reminiscence materials to suit their participants, which can be easily downloaded into their scrapbook. For example, a US-based community health worker supporting a Brazillian PwD in the United States could search the database for reminiscence materials and activities created by community health workers in Brazil. This enables dementia community health workers to share knowledge, provide more inclusive experiences, and decreases the start up costs of launching a new program.

Another example is within the context of an adult day care, an important source of respite for informal caregivers and a place to provide enriching engagement for PwD. Day care centers must assist a diverse group of $\mathrm{PwD}$ where each individual can require personalized care and attention. This heterogeneity creates even more complexity that staff do not have the bandwidth to undertake, particularly in care settings with low staff-to-participant ratios. Managing and monitoring large groups of PwD that vary in stage of dementia, mood fluctuations, and responsiveness can be strenuous. Ubiquitous sensor systems may be helpful to track these changes and alert care staff, which in turn could help reduce their cognitive load and better support their workflow.

\subsection{Design Pitfalls to Avoid}

In addition to these suggestions, we also identified a few key pitfalls to be wary of in dementia technology design to avoid technosolutionism and propagating deficit models of dementia.

Technosolutionism reinforces the deficit model of aging in dementia, by propagating the view that technology can "fix" it, by ignoring key stakeholders and sociotechnical design concepts [61]. To avoid this, dementia technology designers can incorporate critical dementia perspectives, which focus on leveraging strengths in $\mathrm{PwD}$, for instance through embodied expressions of self. In addition, they can incorporate community health perspectives to support the greater ecosystems of care surrounding informal caregivers and PwD, such as community health workers or nursing homes.

Finally, it is important designers consider ways to advance health equity, as this will ultimately play a key role in both the adoption of new technologies as well as their successful use. Here, it can be helpful to be mindful of additional SDoH which impact these factors. These include:

1) Affordability and Cost. Many of those with dementia who live at home face a high rate of poverty [39, 81, 83], and many health systems are resource-limited, especially given the current financial crisis due to the pandemic. Thus, it is important that dementia 
technology designers are mindful of cost when envisioning future systems. In our work, we discuss embodied robotic technology, which have traditionally had high cost. However, as shown by Spoonbot's low cost design and other work [89, 111], robots can be designed to be low cost. Here, it may be helpful to adopt best practices and lessons learned from other areas of HCI, such as from those building technologies for rural health settings and in the developing world [37, 86, 91, 96].

2) Broadband Internet Access. As discussed in recent work by Benda et al., broadband internet access (BIA) is, in itself, a SDoH [10]. This is particularly relevant for the dementia caregiving ecosystem, as over one-third of US-based older adults do not use the internet at all [27]. Thus, it is important to consider technologies that either can operate fully independently of an internet connection, or that provisions are made for providing hotspots to users.

3) Technology Literacy. Older adults tend to have lower levels of technology literacy and familiarity compared to other age groups $[3,27,38]$, which can also affect the success of health interventions. For example, Fields et al. [27] reported a community-based technology intervention study to educate and connect socially-isolated older adults. While the training efforts were successful, the authors found no changes in feelings of loneliness. Technology that is designed to mimic familiar analog technology in design and use may be more effective for this population.

Though these barriers are challenging, they highlight how fundamental principles in HCI, including usability, accessibility, and learnability, are ever more important when designing for older adult caregivers and PwD. In a recent review article, Lindeman et al. [63] offer a helpful conceptual framework that frames design for informal caregivers through a geronotology perspective. They offer technology designers new avenues of research to explore to address health inequities through the design of caregiving technologies.

\subsection{Limitations}

The major limitation of this work is that due to the COVID-19 pandemic, we were unable to work with PwD or informal caregivers. Our partner care facilities were shuttered, leaving many informal caregivers without support and respite resources. This greatly limited the availability of informal caregivers and PwD to participate in research, who were already quite limited pre-pandemic. Furthermore, many dementia families in our community do not have reliable internet access, nor are comfortable using video teleconference, and we did not want to add to their burden.

As such, our paper only presents findings on how professional dementia caregivers approach supporting agency in PwD. We cannot conclude if our findings are effective at supporting agency in PwD without additional work involving PwD. We plan to address this in future work by working with informal caregivers and PwD as soon as it is safe and feasible to do so.

However, we believe, as others in the HCI community do [18, 23, 71], that the perspectives of dementia practitioners can be valuable in the creation of dementia caregiving technologies, so long as one remains mindful of the differences in perspectives and potential for power asymmetries to create design tensions. As our participants were community health workers, we observed great dedication to and key focus on supporting PwD and their informal caregivers. This was reflected across all aspects of how they envisioned the use of technology to support the caregiving process, including supporting the dignity, autonomy, and emotional well-being of PwD and their informal caregivers.

\section{CONCLUSION}

In this paper, we investigated how caregiving support technology can reflect key concepts in dementia community health practice. We identified key takeaways from community practices for informal caregiving, including: community best practices to promote agency in $\mathrm{PwD}$, interactions between emotional well being and caregiving tasks, ways to employ cueing, and roles for embodied technologies. Drawing from our findings, we propose employing embodied cues, such as imitation or action mimicry, as a communication modality that can align technology with community caregiving approaches, potentially promote agency in people with dementia, and relieve caregiver burden.We also suggested new avenues for HCI research to advance health equity by taking a community health perspective in the context of dementia technology design. Addressing complex social issues like health equity through technology design will require interdisciplinary research across the fields of HCI, public health, education, and others. In light of this, our work represents one starting point for future HCI research to gain greater understanding of how technology design can address $\mathrm{SDoH}$ in order to advance health equity.

\section{ACKNOWLEDGMENTS}

We thank Tania Morimoto for her expertise and assistance in creating the prototype. We also thank Vale Gonzales, Amy Abrams, Hee Rin Lee, and Mindy Baker for their invaluable support and insight. Research reported in this paper is supported by the National Science Foundation under Grant No. IIS-1915734.

\section{REFERENCES}

[1] Ridwan Alam, Martha Anderson, Azziza Bankole, and John Lach. 2018. Inferring physical agitation in dementia using smartwatch and sequential behavior models. In 2018 IEEE EMBS International Conference on Biomedical \& Health Informatics (BHI). IEEE, Las Vegas, NV, USA, 170-173.

[2] Zahid Ansari, Norman J Carson, Michael J Ackland, Loretta Vaughan, and Adrian Serraglio. 2003. A public health model of the social determinants of health. Sozial-und Präventivmedizin/Social and Preventive Medicine 48, 4 (2003), 242-251.

[3] Thomas A Arcury, Joanne C Sandberg, Kathryn P Melius, Sara A Quandt, Xiaoyan Leng, Celine Latulipe, David P Miller Jr, D Alden Smith, and Alain G Bertoni. 2020. Older adult internet use and eHealth literacy. Journal of Applied Gerontology 39, 2 (2020), 141-150.

[4] Cathrine Arntzen, Torhild Holthe, and Rita Jentoft. 2016. Tracing the successful incorporation of assistive technology into everyday life for younger people with dementia and family carers. Dementia 15, 4 (2016), 646-662.

[5] AJ Astell, M Ellis, N Alm, R Dye, J Campbell, and G Gowans. 2004. Facilitating communication in dementia with multimedia technology. Brain and Language 91, 1 (2004), 80-81.

[6] Amee Baird and William Forde Thompson. 2018. The impact of music on the self in dementia. Journal of Alzheimer's Disease 61, 3 (2018), 827-841.

[7] Eva Barrett, Megan Burke, Sally Whelan, Adam Santorelli, Barbara Luz Oliveira, Filippo Cavallo, Rose Marie Dröes, Louise Hopper, Ainna Fawcett-Henesy, Franka JM Meiland, et al. 2019. Evaluation of a companion robot for individuals with dementia: quantitative findings of the MARIO project in an Irish residential care setting. Fournal of Gerontological Nursing 45, 7 (2019), 36-45.

[8] Melanie Bayly, Debra Morgan, Amanda Froehlich Chow, Julie Kosteniuk, and Valerie Elliot. 2020. Dementia-Related Education and Support Service Availability, Accessibility, and Use in Rural Areas: Barriers and Solutions. Canadian fournal on Aging/La Revue canadienne du vieillissement 39 (2020), 1-41.

[9] Dror Ben-Zeev, Christopher J Brenner, Mark Begale, Jennifer Duffecy, David C Mohr, and Kim T Mueser. 2014. Feasibility, acceptability, and preliminary efficacy 
of a smartphone intervention for schizophrenia. Schizophrenia bulletin 40, 6 (2014), 1244-1253.

[10] Natalie C Benda, Tiffany C Veinot, Cynthia J Sieck, and Jessica S Ancker. 2020 Broadband Internet Access Is a Social Determinant of Health!

[11] Mark Blythe, Jamie Steane, Jenny Roe, and Caroline Oliver. 2015. Solutionism, the game: design fictions for positive aging. In Proceedings of the 33rd annual ACM conference on human factors in computing systems. Association for Computing Machinery, New York, NY, USA, 3849-3858.

[12] Aikaterini Bourazeri and Simone Stumpf. 2018. Co-designing smart home technology with people with dementia or Parkinson's disease. In Proceedings of the 10th Nordic Conference on Human-Computer Interaction. Association for Computing Machinery, New York, NY, USA, 609-621.

[13] Rens Brankaert, Gail Kenning, Daniel Welsh, Sarah Foley, James Hodge, and David Unbehaun. 2019. Intersections in HCI, design and dementia: inclusivity in participatory approaches. In Companion Publication of the 2019 on Designing Interactive Systems Conference 2019 Companion. Association for Computing Machinery, New York, NY, USA, 357-360.

[14] Virginia Braun and Victoria Clarke. 2012. Thematic analysis. American Psychological Association, 750 First St. NE, Washington, DC 20002-4242.

[15] C-Y Chiao, H-S Wu, and C-Y Hsiao. 2015. Caregiver burden for informal caregivers of patients with dementia: A systematic review. International nursing review 62, 3 (2015), 340-350.

[16] Raymundo Cornejo, Robin Brewer, Caroline Edasis, and Anne Marie Piper. 2016 Vulnerability, sharing, and privacy: Analyzing art therapy for older adults with dementia. In Proceedings of the 19th ACM Conference on Computer-Supported Cooperative Work \& Social Computing. Association for Computing Machinery, New York, NY, USA, 1572-1583.

[17] National Research Council et al. 2010. Informal caregivers in the United States: prevalence, caregiver characteristics, and ability to provide care. National Academies Press (US), 500 Fifth Street, NW Washington, DC.

[18] Yngve Dahl and Dag Svanæs. 2020. Facilitating Democracy: Concerns from Participatory Design with Asymmetric Stakeholder Relations in Health Care. In Proceedings of the 2020 CHI Conference on Human Factors in Computing Systems. Association for Computing Machinery, New York, NY, USA, 1-13.

[19] Jiamin Dai and Karyn Moffatt. 2020. Making Space for Social Sharing: Insights from a Community-Based Social Group for People with Dementia. In Proceeding of the 2020 CHI Conference on Human Factors in Computing Systems. Association for Computing Machinery, New York, NY, USA, 1-13.

[20] Eric Deng, Bilge Mutlu, Maja J Mataric, et al. 2019. Embodiment in Socially Interactive Robots. Foundations and Trends ${ }^{\circledR}$ in Robotics 7, 4 (2019), 251-356.

[21] Margaret Denton, Steven Prus, and Vivienne Walters. 2004. Gender differences in health: a Canadian study of the psychosocial, structural and behavioural determinants of health. Social science \& medicine 58, 12 (2004), 2585-2600.

[22] Peggye Dilworth-Anderson, Heehyul Moon, and María P Aranda. 2020. Dementia Caregiving Research: Expanding and Reframing the Lens of Diversity, Inclusivity, and Intersectionality. The Gerontologist 60, 5 (2020), 797-805.

[23] Emma Dixon and Amanda Lazar. 2020. Approach Matters: Linking Practitioner Approaches to Technology Design for People with Dementia. In Proceedings of the 2020 CHI Conference on Human Factors in Computing Systems. Association for Computing Machinery, New York, NY, USA, 1-15.

[24] Grazia D’Onofrio, Daniele Sancarlo, Francesco Ricciardi, Francesco Panza, Davide Seripa, Filippo Cavallo, Francesco Giuliani, and Antonio Greco. 2017. In formation and communication technologies for the activities of daily living in older patients with dementia: A systematic review. Journal of Alzheimer's Disease 57, 3 (2017), 927-935.

[25] R. el Kaliouby, A. Teeters, and R. W. Picard. 2006. An exploratory socialemotional prosthetic for autism spectrum disorders. In International Workshop on Wearable and Implantable Body Sensor Networks (BSN'06). IEEE, 445 Hoes Lane Piscataway NJ, 2 pp. -4 . https://doi.org/10.1109/BSN.2006.34

[26] Juan Fasola and M Mataric. 2011. Comparing physical and virtual embodiment in a socially assistive robot exercise coach for the elderly. Center for Robotics and Embedded Systems, Los Angeles, CA 2, 2 (2011), 3-32.

[27] Jessica Fields, Anupama Cemballi, Cathy Michalec, Debbie Uchida, Kami Grif fiths, Heather Cardes, Jacqueline Cuellar, Anna Chodos, and Courtney Lyles. 2020. In-Home Technology Training Among Socially Isolated Older Adults: Findings From the Tech Allies Program. Fournal of Applied Gerontology 0, 0 (03 2020), 073346482091002. https://doi.org/10.1177/0733464820910028

[28] Sarah Foley, John McCarthy, and Nadia Pantidi. 2019. The Struggle for Recognition in Advanced Dementia: Implications for Experience-Centered Design. ACM Transactions on Computer-Human Interaction (TOCHI) 26, 6 (2019), 1-29.

[29] Sarah Foley, Nadia Pantidi, and John McCarthy. 2019. Care and design: An ethnography of mutual recognition in the context of advanced dementia. In Proceedings of the 2019 CHI Conference on Human Factors in Computing Systems. Association for Computing Machinery, New York, NY, USA, 1-15.

[30] Sarah Foley and Daniel Welsh. 2020. Bridging the Gap: Design for Intergenerational Engagement in Dementia Care. In HCI and Design in the Context of Dementia. Springer, $11 \mathrm{~W}$ 42nd St \#15, New York, NY 10036, 255-265.

[31] Sarah Foley, Daniel Welsh, Nadia Pantidi, Kellie Morrissey, Tom Nappey, and John McCarthy. 2019. Printer Pals: Experience-centered design to support agency for people with dementia. In Proceedings of the 2019 CHI Conference on Human Factors in Computing Systems. Association for Computing Machinery, New York, NY, USA, 1-13.

[32] Pin Sym Foong, Shengdong Zhao, Kelsey Carlson, and Zhe Liu. 2017. VITA: Towards Supporting Volunteer Interactions with Long-Term Care Residents with Dementia. In Proceedings of the 2017 CHI Conference on Human Factors in Computing Systems. Association for Computing Machinery, New York, NY, USA, 6195-6207.

[33] Centers for Disease Control and Prevention. 2020. Social Determinants of Health. https://www.cdc.gov/socialdeterminants/about.html. Accessed: 2020-09-17.

[34] Mary Galvin. 2016. Supporting positive interactions within dementia relationships of care. Ph.D. Dissertation. University College Cork.

[35] Montserrat Gea-Sánchez, Denise Gastaldo, Fidel Molina-Luque, and Laura OteroGarcía. 2017. Access and utilisation of social and health services as a social determinant of health: the case of undocumented Latin A merican immigrant women working in Leida. Health \& social care in the community 25, 2 (2017), 424-434.

[36] Moojan Ghafurian, Jesse Hoey, Daniel Tchorni, Annika Ang, Mallorie Tam, and Julie M Robillard. 2020. Emotional Alignment Between Older Adults and Online Personalities: Implications for Assistive Technologies. (2020). (in press).

[37] Jean Hardy, Susan Wyche, and Tiffany Veinot. 2019. Rural HCI research: Definitions, distinctions, methods, and opportunities. Proceedings of the ACM on Human-Computer Interaction 3, CSCW (2019), 1-33.

[38] Eszter Hargittai, Anne Marie Piper, and Meredith Ringel Morris. 2019. From internet access to internet skills: digital inequality among older adults. Universal Access in the Information Society 18, 4 (2019), 881-890.

[39] Krista L Harrison, Christine S Ritchie, Kanan Patel, Lauren J Hunt, Kenneth E Covinsky, Kristine Yaffe, and Alexander K Smith. 2019. Care settings and clinical characteristics of older adults with moderately severe dementia. Fournal of the American Geriatrics Society 67, 9 (2019), 1907-1912.

[40] Theresa A Harvath, Jennifer M Mongoven, Julie T Bidwell, Fawn A Cothran, Kathryn E Sexson, Diana J Mason, and Kathleen Buckwalter. 2020. Research priorities in family caregiving: Process and outcomes of a conference on familycentered care across the trajectory of serious illness. The Gerontologist 60 , Supplement_1 (2020), S5-S13.

[41] James Hodge, Madeline Balaam, Sandra Hastings, and Kellie Morrissey. 2018. Exploring the design of tailored virtual reality experiences for people with dementia. In Proceedings of the 2018 CHI Conference on Human Factors in Computing Systems. Association for Computing Machinery, New York, NY, USA, $1-13$.

[42] Maarten Houben, Rens Brankaert, Saskia Bakker, Gail Kenning, Inge Bongers, and Berry Eggen. 2019. Foregrounding everyday sounds in dementia. In Proceedings of the 2019 on Designing Interactive Systems Conference. Association for Computing Machinery, New York, NY, USA, 71-83.

[43] Maarten Houben, Rens Brankaert, Saskia Bakker, Gail Kenning, Inge Bongers, and Berry Eggen. 2020. The role of everyday sounds in advanced dementia care. In Proceedings of the 2020 CHI Conference on Human Factors in Computing Systems. Association for Computing Machinery, New York, NY, USA, 1-14.

[44] Maarten Houben, Benjamin Lehn, Noa van den Brink, Sabeth Diks, Jasmijn Verhoef, and Rens Brankaert. 2020. Turnaround: Exploring Care Relations in Dementia Through Design. In Extended Abstracts of the 2020 CHI Conference on Human Factors in Computing Systems. Association for Computing Machinery, New York, NY, USA, 1-8.

[45] Wijnand IJsselsteijn, Ans Tummers-Heemels, and Rens Brankaert. 2020. Warm Technology: A Novel Perspective on Design for and with People Living with Dementia. In HCI and Design in the Context of Dementia. Springer, $11 \mathrm{~W} 42 \mathrm{nd}$ St \#15, New York, NY 10036, 33-47.

[46] Sooyeon Jeong. 2017. The impact of social robots on young patients' socioemotional wellbeing in a pediatric inpatient care context. Ph.D. Dissertation. Massachusetts Institute of Technology.

[47] Sooyeon Jeong, Cynthia Breazeal, Deirdre Logan, and Peter Weinstock. 2017. Huggable: Impact of embodiment on promoting verbal and physical engagement for young pediatric inpatients. In 2017 26th IEEE international symposium on robot and human interactive communication (RO-MAN). IEEE, 445 Hoes Lane Piscataway NJ, 121-126.

[48] Marije Kanis, Saskia Robben, Judith Hagen, Anne Bimmerman, Natasja Wagelaar, and Ben Kröse. 2013. Sensor monitoring in the home: giving voice to elderly people. In 2013 7th International Conference on Pervasive Computing Technologies for Healthcare and Workshops. IEEE, 445 Hoes Lane Piscataway NJ, 97-100.

[49] Vicky Karkou and Bonnie Meekums. 2017. Dance movement therapy for dementia. Cochrane Database of Systematic Reviews 2, 2 (2017)

[50] Sohyun Kim, Clarissa Shaw, Kristine N Williams, and Maria Hein. 2019. Typology of technology-supported dementia care interventions from an in-home telehealth trial. Western journal of nursing research 41, 12 (2019), 1724-1746.

[51] Pia Kontos and Wendy Martin. 2013. Embodiment and dementia: Exploring critical narratives of selfhood, surveillance, and dementia care. Dementia 12, 3 (2013), 288-302. 
[52] Pia C Kontos. 2005. Embodied selfhood in Alzheimer's disease: Rethinking person-centred care. Dementia 4, 4 (2005), 553-570.

[53] Bon Mi Koo and Lisa M Vizer. 2019. Examining Mobile Technologies to Support Older Adults With Dementia Through the Lens of Personhood and Human Needs: Scoping Review. FMIR mHealth and uHealth 7, 11 (2019), e15122.

[54] Stephen C Kramer, Erika Friedmann, and Penny L Bernstein. 2009. Comparison of the effect of human interaction, animal-assisted therapy, and AIBO-assisted therapy on long-term care residents with dementia. Anthrozoös 22, 1 (2009), 43-57.

[55] Alyssa Kubota, Emma I. C. Peterson, Vaishali Rajendren, Hadas Kress-Gazit, and Laurel D. Riek. 2020. JESSIE: Synthesizing Social Robot Behaviors for Personalized Neurorehabilitation and Beyond. In Proceedings of the 2020 ACM/IEEE International Conference on Human-Robot Interaction (Cambridge, United Kingdom) (HRI '20). Association for Computing Machinery, New York, NY, USA 121-130. https://doi.org/10.1145/3319502.3374836

[56] A. Kubota and L.D. Riek. 2021. Methods for Robot Behavior Adaptation for Cognitive Neurorehabilitation. (2021). (in press).

[57] Amanda Lazar, Caroline Edasis, and Anne Marie Piper. 2017. A Critical Lens on Dementia and Design in HCI. In Proceedings of the 2017 CHI Conference on Human Factors in Computing Systems (Denver, Colorado, USA) (CHI '17). Association for Computing Machinery, New York, NY, USA, 2175-2188. https: //doi.org/10.1145/3025453.3025522

[58] Amanda Lazar, Jessica L Feuston, Caroline Edasis, and Anne Marie Piper. 2018 Making as expression: Informing design with people with complex communication needs through art therapy. In Proceedings of the 2018 CHI Conference on Human Factors in Computing Systems. Association for Computing Machinery, New York, NY, USA, 1-16.

[59] Amanda Lazar, Hilaire Thompson, and George Demiris. 2014. A systematic review of the use of technology for reminiscence therapy. Health education \& behavior 41, 1_suppl (2014), 51S-61S.

[60] Amanda Lazar, Austin L Toombs, Kellie Morrissey, Gail Kenning, Jennifer Boger, and Rens Brankaert. 2018. Hcixdementia workshop: Engaging people living with dementia. In Extended Abstracts of the 2018 CHI Conference on Human Factors in Computing Systems. Association for Computing Machinery, New York, NY, USA, 1-7.

[61] Hee Rin Lee and Laurel D Riek. 2018. Reframing assistive robots to promote successful aging. ACM Transactions on Human-Robot Interaction (THRI) 7, 1 (2018), 1-23.

[62] Jean-Frederic Levesque, Mark F Harris, and Grant Russell. 2013. Patient-centred access to health care: conceptualising access at the interface of health systems and populations. International journal for equity in health 12, 1 (2013), 18.

[63] David A Lindeman, Katherine K Kim, Caroline Gladstone, and Ester Carolina Apesoa-Varano. 2020. Technology and caregiving: Emerging interventions and directions for research. The Gerontologist 60, Supplement_1 (2020), S41-S49.

[64] Klara Lorenz, Paul P Freddolino, Adelina Comas-Herrera, Martin Knapp, and Jacqueline Damant. 2019. Technology-based tools and services for people with dementia and carers: Mapping technology onto the dementia care pathway. Dementia 18, 2 (2019), 725-741.

[65] Michael Marmot. 2005. Social determinants of health inequalities. The lancet 365, 9464 (2005), 1099-1104.

[66] Patrizia Marti, Margherita Bacigalupo, Leonardo Giusti, Claudio Mennecozzi, and Takanori Shibata. 2006. Socially assistive robotics in the treatment of behavioural and psychological symptoms of dementia. In The First IEEE/RASEMBS International Conference on Biomedical Robotics and Biomechatronics, 2006. BioRob 2006. IEEE, 445 Hoes Lane Piscataway NJ, 483-488.

[67] Pekka Martikainen, Mel Bartley, and Eero Lahelma. 2002. Psychosocial determinants of health in social epidemiology.

[68] Roisin McNaney, John Vines, Jamie Mercer, Leon Mexter, Daniel Welsh, and Tony Young. 2017. DemYouth: Co-designing and enacting tools to support young people's engagement with people with dementia. In Proceedings of the 2017 CHI Conference on Human Factors in Computing Systems. Association for Computing Machinery, New York, NY, USA, 1313-1325.

[69] Herlind Megges, Silka Dawn Freiesleben, Natalie Jankowski, Brigitte Haas, and Oliver Peters. 2017. Technology for home dementia care: A prototype locating system put to the test. Alzheimer's \& Dementia: Translational Research \& Clinical Interventions 3, 3 (2017), 332-338.

[70] Sanika Moharana, Alejandro E Panduro, Hee Rin Lee, and Laurel D Riek. 2019. Robots for joy, robots for sorrow: community based robot design for dementia caregivers. In 2019 14th ACM/IEEE International Conference on Human-Robot Interaction (HRI). IEEE, 445 Hoes Lane Piscataway NJ, 458-467.

[71] Argyro Moraiti, Vero Vanden Abeele, Erwin Vanroye, and Luc Geurts. 2015. Empowering occupational therapists with a DIY-toolkit for smart soft objects. In Proceedings of the Ninth International Conference on Tangible, Embedded, and Embodied Interaction. Association for Computing Machinery, New York, NY, USA, 387-394.

[72] Debra G Morgan, Julie G Kosteniuk, Norma J Stewart, Megan E O'Connell, Andrew Kirk, Margaret Crossley, Vanina Dal Bello-Haas, Dorothy Forbes, and Anthea Innes. 2015. Availability and primary health care orientation of dementiarelated services in rural Saskatchewan, Canada. Home Health Care Services
Quarterly 34, 3-4 (2015), 137-158.

[73] Kellie Morrissey, Amanda Lazar, Jennifer Boger, and Austin Toombs. 2017. Hcixdementia workshop: The role of technology and design in dementia. In Proceedings of the 2017 CHI Conference Extended Abstracts on Human Factors in Computing Systems. Association for Computing Machinery, New York, NY, USA, 484-491.

[74] Kellie Morrissey, John McCarthy, and Nadia Pantidi. 2017. The value of experience-centred design approaches in dementia research contexts. In Proceedings of the 2017 CHI Conference on Human Factors in Computing Systems. Association for Computing Machinery, New York, NY, USA, 1326-1338.

[75] Kellie Morrissey, Gavin Wood, David Green, Nadia Pantidi, and John McCarthy. 2016. 'I'm a Rambler, I'm a Gambler, I'm a Long Way from Home': The Place of Props, Music, and Design in Dementia Care. In Proceedings of the 2016 ACM Conference on Designing Interactive Systems (Brisbane, QLD, Australia) (DIS '16). Association for Computing Machinery, New York, NY, USA, 1008-1020. https://doi.org/10.1145/2901790.2901798

[76] Wendy Moyle, Cindy J Jones, Jenny E Murfield, Lukman Thalib, Elizabeth RA Beattie, David KH Shum, Siobhan T O’Dwyer, M Cindy Mervin, and Brian M Draper. 2017. Use of a robotic seal as a therapeutic tool to improve dementia symptoms: a cluster-randomized controlled trial. Fournal of the American Medical Directors Association 18, 9 (2017), 766-773.

[77] Inbal Nahum-Shani, Shawna N Smith, Bonnie J Spring, Linda M Collins, Katie Witkiewitz, Ambuj Tewari, and Susan A Murphy. 2018. Just-in-time adaptive interventions (JITAIs) in mobile health: key components and design principles for ongoing health behavior support. Annals of Behavioral Medicine 52, 6 (2018), 446-462.

[78] Renee Noortman, Britta F Schulte, Paul Marshall, Saskia Bakker, and Anna L Cox. 2019. HawkEye-Deploying a Design Fiction Probe. In Proceedings of the 2019 CHI Conference on Human Factors in Computing Systems. Association for Computing Machinery, New York, NY, USA, 1-14.

[79] National Academies of Sciences Engineering, Medicine, et al. 2016. Families caring for an aging America. National Academies Press, 500 Fifth St., N.W. Washington, D.C 20001.

[80] World Health Organization et al. 2010. A conceptual framework for action on the social determinants of health.

[81] World Health Organization et al. 2017. Global action plan on the public health response to dementia 2017-2025.

[82] Marcia G Ory, Richard R Hoffman III, Jennifer L Yee, Sharon Tennstedt, and Richard Schulz. 1999. Prevalence and impact of caregiving: A detailed comparison between dementia and nondementia caregivers. The Gerontologist 39, 2 (1999), 177-186.

[83] Anne Margriet Pot, Dolores Gallagher-Thompson, Lily D Xiao, Bernadette M Willemse, Iris Rosier, Kala M Mehta, Diana Zandi, Tarun Dua, and iSupport development team. 2019. iSupport: a WHO global online intervention for informal caregivers of people with dementia. World Psychiatry 18, 3 (2019), 365-366.

[84] Gede Pramana, Bambang Parmanto, Philip C Kendall, and Jennifer S Silk. 2014. The SmartCAT: an m-health platform for ecological momentary intervention in child anxiety treatment. Telemedicine and e-Health 20, 5 (2014), 419-427.

[85] Solveig Magnus Reindal. 2008. A social relational model of disability: a theoretical framework for special needs education? European fournal of Special Needs Education 23, 2 (2008), 135-146.

[86] Christian Remy, Oliver Bates, Alan Dix, Vanessa Thomas, Mike Hazas, Adrian Friday, and Elaine M Huang. 2018. Evaluation beyond usability: Validating sustainable HCI research. In Proceedings of the 2018 CHI Conference on Human Factors in Computing Systems. Association for Computing Machinery, New York, NY, USA, 1-14.

[87] Laurel D Riek. 2016. Robotics technology in mental health care. In Artificial intelligence in behavioral and mental health care. Elsevier, Radarweg 29, 1043 NX Amsterdam, The Netherlands, 185-203.

[88] Laurel D. Riek. 2017. Healthcare Robotics. Commun. ACM 60, 11 (Oct. 2017), 68-78. https://doi.org/10.1145/3127874

[89] M. Rubenstein, C. Ahler, and R. Nagpal. 2012. Kilobot: A low cost scalable robot system for collective behaviors. In 2012 IEEE International Conference on Robotics and Automation. IEEE, 445 Hoes Lane Piscataway NJ, 3293-3298. https://doi.org/10.1109/ICRA.2012.6224638

[90] Selma Šabanović, Casey C Bennett, Wan-Ling Chang, and Lesa Huber. 2013. PARO robot affects diverse interaction modalities in group sensory therapy for older adults with dementia. In 2013 IEEE 13th international conference on rehabilitation robotics (ICORR). IEEE, 445 Hoes Lane Piscataway NJ, 1-6.

[91] Ari Schlesinger, W Keith Edwards, and Rebecca E Grinter. 2017. Intersectional HCI: Engaging identity through gender, race, and class. In Proceedings of the 2017 CHI conference on human factors in computing systems. Association for Computing Machinery, New York, NY, USA, 5412-5427.

[92] Britta F Schulte. 2016. Using design fiction to reflect on autonomy in smart technology for people living with dementia. In Proceedings of the 2016 ACM International foint Conference on Pervasive and Ubiquitous Computing: Adjunct. Association for Computing Machinery, New York, NY, USA, 1110-1113. 
[93] Britta F Schulte, Paul Marshall, and Anna L Cox. 2016. Homes for life: a design fiction probe. In Proceedings of the 9th nordic conference on human-computer interaction. Association for Computing Machinery, New York, NY, USA, 1-10.

[94] Richard Schulz and Lynn M Martire. 2004. Family caregiving of persons with dementia: prevalence, health effects, and support strategies. The American journal of geriatric psychiatry 12, 3 (2004), 240-249.

[95] Majid Shishehgar, Donald Kerr, and Jacqueline Blake. 2018. A systematic review of research into how robotic technology can help older people. Smart Health 7 (2018), 1-18

[96] Katie Siek, Tiffany Veinot, and Beth Mynatt. 2019. Research Opportunities in Sociotechnical Interventions for Health Disparity Reduction. arXiv: 1908.01035 [cs.CY]

[97] Vimal Sriram, Crispin Jenkinson, and Michele Peters. 2019. Informal carers experience of assistive technology use in dementia care at home: a systematic review. BMC geriatrics 19, 1 (2019), 160.

[98] Sandra Suijkerbuijk, Henk Herman Nap, Lotte Cornelisse, Wijnand A IJsselsteijn, Yvonne AW De Kort, and Mirella Minkman. 2019. Active involvement of people with dementia: a systematic review of studies developing supportive technologies. Fournal of Alzheimer's Disease 69, 4 (2019), 1041-1065.

[99] Leila Takayama, Victoria Groom, and Clifford Nass. 2009. I'm sorry, Dave: i'm afraid i won't do that: social aspects of human-agent conflict. In Proceedings of the SIGCHI Conference on Human Factors in Computing Systems. Association for Computing Machinery, New York, NY, USA, 2099-2108.

[100] Toshiyo Tamura, Satomi Yonemitsu, Akiko Itoh, Daisuke Oikawa, Akiko Kawakami, Yuji Higashi, Toshiro Fujimooto, and Kazuki Nakajima. 2004. Is an entertainment robot useful in the care of elderly people with severe dementia? The fournals of Gerontology Series A: Biological Sciences and Medical Sciences 59 , 1 (2004), M83-M85.

[101] Angelique Taylor, Hee Rin Lee, Alyssa Kubota, and Laurel D. Riek. 2019. Coordinating Clinical Teams: Using Robots to Empower Nurses to Stop the Line. Proc. ACM Hum.-Comput. Interact. 3, CSCW, Article 221 (Nov. 2019), 30 pages. https://doi.org/10.1145/3359323

[102] Hsiu-Hsin Tsai, Ching-Yu Cheng, Wann-Yun Shieh, and Yue-Cune Chang. 2020. Effects of a smartphone-based videoconferencing program for older nursing home residents on depression, loneliness, and quality of life: a quasiexperimental study. BMC geriatrics 20, 1 (2020), 27.

[103] George E Vaillant. 2008. Aging well: Surprising guideposts to a happier life from the landmark study of adult development. Hachette UK, Carmelite House, 50 Victoria Embankment, London EC4Y 0DZ, United Kingdom.

[104] Sridhar Vaitheswaran, Monisha Lakshminarayanan, Vaishnavi Ramanujam, Subashini Sargunan, and Shreenila Venkatesan. 2020. Experiences and Needs of Caregivers of Persons With Dementia in India During the COVID-19 Pandemic-A Qualitative Study. The American fournal of Geriatric Psychiatry 28, 11 (2020), 1185-1194.

[105] Ryan Van Patten, Amber V Keller, Jacqueline E Maye, Dilip V Jeste, Colin Depp Laurel D Riek, and Elizabeth W Twamley. 2020. Home-Based Cognitively Assistive Robots: Maximizing Cognitive Functioning and Maintaining Independence in Older Adults Without Dementia. Clinical Interventions in Aging 15 (2020), 1129 .

[106] Tiffany C Veinot, Jessica S Ancker, Heather Cole-Lewis, Elizabeth D Mynatt Andrea G Parker, Katie A Siek, and Lena Mamykina. 2019. Leveling up: on the potential of upstream health informatics interventions to enhance health equity. Medical care 57 (2019), S108-S114.
[107] Ellen Verbakel, Stian Tamlagsrønning, Lizzy Winstone, Erlend L Fjær, and Terje A Eikemo. 2017. Informal care in Europe: findings from the European Social Survey (2014) special module on the social determinants of health. The European fournal of Public Health 27, suppl 1 (2017), 90-95.

[108] Annemiek C Vink, Manon S Bruinsma, and Rob JPM Scholten. 2003. Music therapy for people with dementia.

[109] Kazuyoshi Wada, Takanori Shibata, Toshimitsu Musha, and Shin Kimura. 2008. Robot therapy for elders affected by dementia. IEEE Engineering in medicine and biology magazine 27, 4 (2008), 53-60.

[110] Jayne Wallace, Peter C Wright, John McCarthy, David Philip Green, James Thomas, and Patrick Olivier. 2013. A design-led inquiry into personhood in dementia. In Proceedings of the SIGCHI Conference on Human Factors in Com puting Systems. Association for Computing Machinery, New York, NY, USA 2617-2626.

[111] Jerry B Weinberg, Xudong Yu, et al. 2003. Robotics in education: Low-cost platforms for teaching integrated systems. IEEE Robotics \& automation magazine 10, 2 (2003), 4-6.

[112] Daniel Welsh, Kellie Morrissey, Sarah Foley, Roisin McNaney, Christos Salis, John McCarthy, and John Vines. 2018. Ticket to talk: Supporting conversation between young people and people with dementia through digital media. In proceedings of the 2018 CHI conference on human factors in computing systems. Association for Computing Machinery, New York, NY, USA, 1-14.

[113] Christian Werner, George P Moustris, Costas S Tzafestas, and Klaus Hauer. 2018. User-oriented evaluation of a robotic rollator that provides navigation assistance in frail older adults with and without cognitive impairment. Gerontology 64, 3 (2018), 278-290.

[114] Andrea Wilkinson, Vishuda Charoenkitkarn, Judy O'Neill, Marc Kanik, and Mark Chignell. 2017. Journeys to engagement: Ambient activity technologies for people living with dementia. In Proceedings of the 26th International Conference on World Wide Web Companion. Association for Computing Machinery, New York, NY, USA, 1103-1110.

[115] Richard G Wilkinson and Michael Marmot. 2003. Social determinants of health: the solid facts. World Health Organization, World Health Organization 20 Avenue Appia 1211 Geneva 27 Switzerland.

[116] Kristine Williams, Diane Blyler, Eric D Vidoni, Clarissa Shaw, JoEllen Wurth, Denise Seabold, Yelena Perkhounkova, and Angela Van Sciver. 2018. A randomized trial using telehealth technology to link caregivers with dementia care experts for in-home caregiving support: FamTechCare protocol. Research in nursing \& health 41, 3 (2018), 219-227.

[117] Ryuji Yamazaki, Shuichi Nishio, Kohei Ogawa, and Hiroshi Ishigur. 2012. Teleoperated android as an embodied communication medium: A case study with demented elderlies in a care facility. In 2012 IEEE RO-MAN: The 21st IEEE International Symposium on Robot and Human Interactive Communication. IEEE, 445 Hoes Lane Piscataway NJ, 1066-1071.

[118] Heather M Young, Janice F Bell, Robin L Whitney, Ronit A Ridberg, Sarah C Reed, and Peter P Vitaliano. 2020. Social Determinants of Health: Underreported Heterogeneity in Systematic Reviews of Caregiver Interventions. The Gerontologist 60, Supplement 1 (2020), S14-S28.

[119] Kara Zivin, Ananda Sen, Melissa A Plegue, Matthew L Maciejewski, Michelle L Segar, Mona AuYoung, Erin M Miller, Carol A Janney, Donna M Zulman, and Caroline R Richardson. 2017. Comparative effectiveness of wellness programs: Impact of incentives on healthcare costs for obese enrollees. American journal of preventive medicine 52, 3 (2017), 347-352. 